\title{
MECHANICS OF MAGNETIC FLUID ACTIVE ELEMENT IN STRONG MAGNETIC FIELD
}

\author{
Polunin V.M. ${ }^{1}$, Ryapolov P.A. ${ }^{1}$, Sheldeshova E.V. ${ }^{1}$ \\ ${ }^{1}$ Southwest State University, Kursk, Russia
}

\begin{abstract}
Measurements and theoretical analysis of the processes of static displacement and oscillations of the magnetic fluid column confined by magnetic levitation in a strong magnetic field in a horizontally placed tube are carried out. The calculations of the saturation magnetization, made on the basis of the obtained results of the displacement and the oscillation frequency for the sample of the magnetic fluid under study, are in good agreement with the experimental data. The described technique is of interest when studying saturation magnetization, magnetophoresis, aggregation of nanoparticles and their temporal dependence in magnetic colloids.
\end{abstract}

\section{INTRODUCTION}

The design of many devices using magnetic fluids MF is based on the impact of inhomogeneous magnetic field, i.e., ponderomotive force, on the drop of magnetic colloid; this can be observed while the drop displaces from equilibrium position. Such devices can be called oscillatory systems, wherein MF acts as an inertialviscous element [1-4]. The most common oscillation systems based on nanodispersed MFs, such as controlled shock absorbers and vibration damping complexes, acoustic dynamics with the use of MF, are based on linear vibrations of MF elastic elements on the basis. Therefore, of particular interest are measurements of the elastic-magnetic parameters of a magnetic fluid held by magnetic levitation, in which linear movements of the MF sample are tracked.

\section{EXPERIMENTAL SETUP}

To measure MF-column displacement in a strong magnetic field under hydrostatic pressure an experimental setup, the diagram of which is presented in figure 1 was designed.

In the experiment, we used a laboratory electromagnet FL-1 with a diameter of the pole tips equal to $100 \mathrm{~mm}$. The construction of the electromagnet FL-1 and its specifications are given in [5]. The transparent tube 1 made of plexiglas with the internal diameter $d=12 \mathrm{~mm}$ is fixed between the pole tips. The tube axis passes horizontally through the centre of the pole gap and is parallel to the surface of the pole tips. When the magnetic field intensity is $\geq 100 \mathrm{kA} / \mathrm{m}$, the tube is filled with a magnetic fluid 2, which is captured by the field and holds in the region of high magnetic field. The position of the tube is fixed with the holding device. In the initial state, MF-column is symmetric with respect to the pole centre. One end of the tube using a silicone tube 3 is connected with the U-shaped tube 4 filled with distilled water. The U-shaped tube elbows are of 800 $\mathrm{mm}$, its internal diameter is $16 \mathrm{~mm}$. The difference in water levels $\Delta \mathrm{h}$ in the tube elbows is determined using cathetometer.

To measure MF-column displacement under hydrostatic pressure $-\Delta z$ on the same plane with the horizontal axial section of the tube the reference mark is made as it is shown dashed in figure 1; a camera was placed above the whole system to record data of MF-column boundary displacement. MF-column moves to equilibrium state due to the resultant action of ponderomotive force and hydrostatic pressure.

The diagram of the experimental setup for measuring MF-column oscillation frequency $v$ is shown in Figure 2. There are some new parts in this setup in comparison with the setup shown in Figure 1. The piston 3 is used to drive oscillations. There is a fine air gap between the piston and magnetic fluid. The oscillation indicator, the inductance coil 4 is mounted in the gap between the tube and the pole tip at the level of MF-column free surface. The inductance coil has 5,000 turns of copper wire with a diameter of $0.071 \mathrm{~mm}$ wound around the Plexiglas spool. The signal from the oscillation indicator comes to Selective Nanovoltmeter type 2375 . After amplification, the signal is transmitted to GwInstek GOS-72074 oscillograph 6 , and then to a computer (not shown in Figure 2) for further processing.

The required value of the magnetic field intensity $\mathrm{H}$ in the pole gap of the electromagnet FL-1 is reached by means of two series-connected direct current sources QJE QJ6030S and is registered by Aktakom ATE-8702 teslameter (not shown in Figures 1 and 2).

\footnotetext{
Corresponding author: r-piter@yandex.ru
} 
The experimental dependence of magnetic field intensity (to be more precise, its component $\mathrm{Hx}$ ) on the $\mathrm{z}$ coordinate along the $\mathrm{OZ}$ axis extending from the centre of the pole gap parallel to the surface of the pole tips were measured. The measurements were carried out in the range of magnetic field intensity from $100 \mathrm{kA} / \mathrm{m}$ to $900 \mathrm{kA} / \mathrm{m}$ in increments of $50 \mathrm{kA} / \mathrm{m}$. The teslameter probe moves over the distance of $90 \mathrm{~mm}$ using cathetometer. The probe position at the distances from 0 $\mathrm{mm}$ to $40 \mathrm{~mm}$ and from $70 \mathrm{~mm}$ to $90 \mathrm{~mm}$ is set at a pitch of $10 \mathrm{~mm}$, and at the distance of $40 \mathrm{~mm}$ to $70 \mathrm{~mm}$ it is set at a pitch of $2 \mathrm{~mm}$ is fixed with an accuracy of 0.01 $\mathrm{mm}$. The values of the magnetic field intensity peculiar to MF-column top and bottom are marked with the

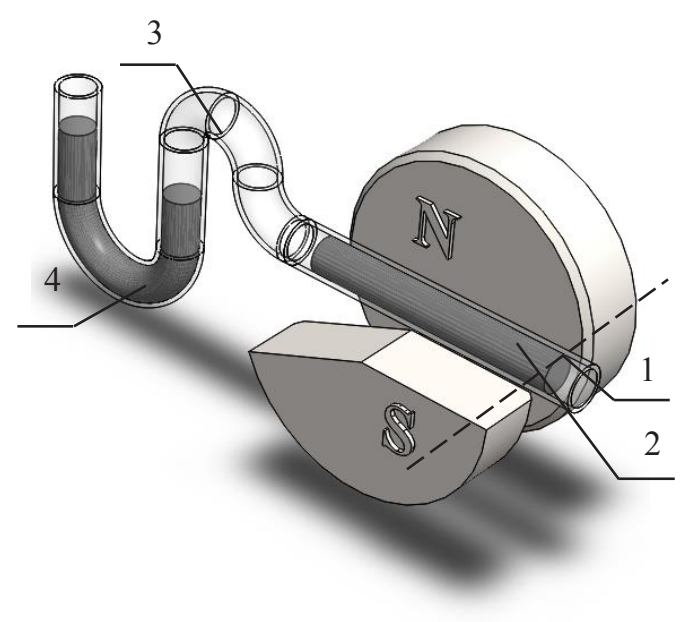

Fig. 1. Static experimental setup diagram

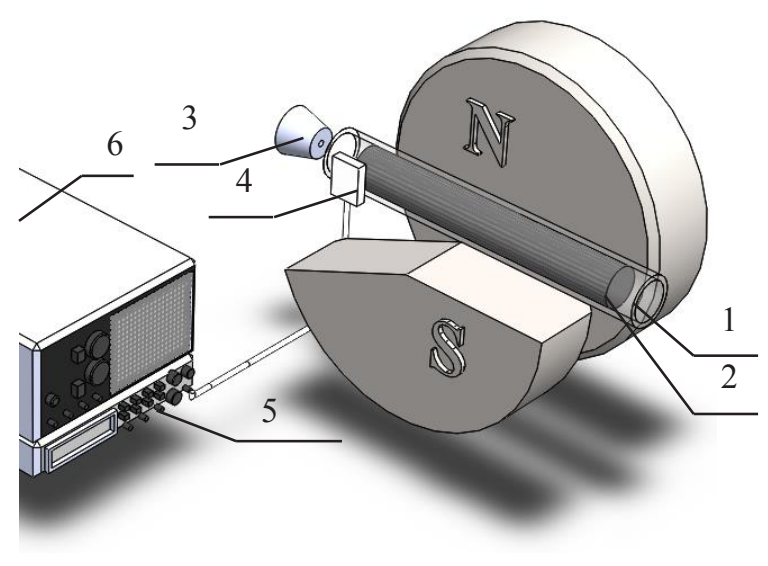

Fig. 2. Dynamic experimental setup diagram

dashed line. A significant feature of the obtained dependencies $\operatorname{Hx}(\mathrm{z})$ is the presence of a linear segment on the curves at the level of $\mathrm{z}=57.5 \mathrm{~mm}$, which allows us to take the magnetic field intensity gradient at this segment as follows: $\Delta H_{x} / \Delta z=$ const

The measurements of physical quantities $v, \Delta \mathrm{h}$, and $\Delta \mathrm{z}$ are made at fixed magnetic field parameters. Table 1 shows the used combination of magnetic field parameters: $\mathrm{H}_{0}$, which is magnetic field intensity in the centre between the poles of the electromagnet, $\mathrm{H}^{*}$, which is magnetic field intensity, and $\Delta \mathrm{Hx} / \Delta \mathrm{z}$, which is the magnetic field intensity gradient.

Table 1

\begin{tabular}{|c|c|c|}
\hline $\begin{array}{c}\mathrm{H}_{0}, \\
\mathrm{kA} / \mathrm{m}\end{array}$ & $\begin{array}{c}\mathrm{H}_{*}, \\
\mathrm{kA} / \mathrm{m}\end{array}$ & $\begin{array}{c}\Delta \mathrm{H}_{\mathrm{x}} / \Delta \mathrm{z}, \\
\mathrm{MA} / \mathrm{m}^{2}\end{array}$ \\
\hline 200 & 156 & 3.42 \\
\hline 300 & 236 & 5.13 \\
\hline 400 & 313 & 6.95 \\
\hline 500 & 391 & 8.7 \\
\hline 600 & 469 & 10.5 \\
\hline 700 & 540 & 12.4 \\
\hline 800 & 619 & 14.2 \\
\hline 900 & 686 & 15.9 \\
\hline
\end{tabular}

In a strong and inhomogeneous magnetic field, MFcolumn takes a form close to a cylindrical one; in the conducted experiment, the distance between the bases of the cylinder is $115 \mathrm{~mm}$.

\section{THEORY OF MF-COLUMN ELASTOMAGNETIC PROPERTIES}

The computational pattern introduced for solving the task is shown in Figure 3. It is assumed that the MF left and right free surfaces are on the linear segments of the magnetic field decrease.

In the view of the assumptions concerning the tube 'thinness', magnetic field at the points of the MF free surface is tangential to it, i.e. it has only the tangential component $\mathrm{Hx}$ and the magnetic field intensity gradient is directed perpendicular to the surface along the $\mathrm{OZ}$ axis to its beginning. Under the action of the differential pressure on the bases of the MF-column 2 with the cross section $\mathrm{S}$ and the length $\mathrm{b}$, a small mass center shift equal to $\delta z$ occurs, which is accompanied by the $S \cdot \delta z$ volume gain of the fluid at the point with the coordinate $\mathrm{z}=\mathrm{b} / 2$ and decrease of its volume at the same value at the point $\mathrm{z}=-\mathrm{b} / 2$.

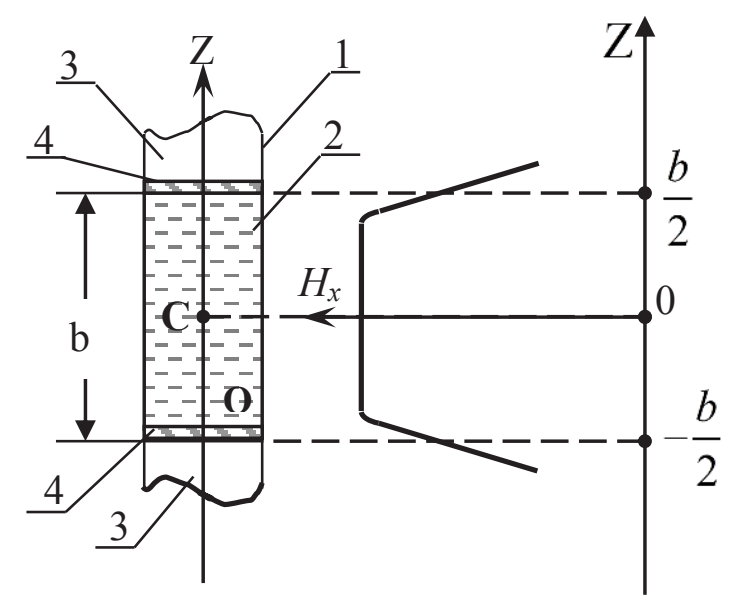

Figure 3. The computational pattern 
For the elasticity coefficients of this system, the following values of the elasticity coefficients were obtained in [6]:

For the static experiment the hydrostatic coefficient determined by the water pressures in the U-shaped manometer:

$$
k_{s}=\pi d^{2} \rho_{w} g \Delta h / 4 \Delta z,
$$

where $\rho_{w}$ is the water density.

The ponderomotive coefficient is determined by the magnetic fluid displacement from the equilibrium position:

$$
k_{p}=\mu_{0} \frac{\pi d^{2}}{2}\left(M_{x} \frac{\partial H_{x}}{\partial z}\right)_{z=b / 2},
$$

For the dynamic experiment the coefficient is determined from the oscillation frequency:

$$
k_{d \omega}=\omega^{2} \pi \rho b d^{2} / 4
$$

where $\rho$ is the MF density.

The ponderomotive coefficient is determined by the magnetic fluid displacement from the equilibrium position with addition to viscous friction:

$$
k_{d}=\mu_{0} \frac{\pi d^{2}}{2}\left(M_{x} \frac{\partial H_{x}}{\partial z}\right)_{z=b / 2}-\frac{\pi^{2}}{2 \sqrt{2}} \cdot b d \sqrt{\omega^{3} \eta \rho}
$$

We studied a sample of finely dispersed magnetitebased magnetic fluid $-\mathrm{Fe}_{3} \mathrm{O}_{4}$ which is stabilized by a surfactant - oleic acid $\mathrm{C}_{8} \mathrm{H}_{17} \mathrm{CH}=\mathrm{CH}\left(\mathrm{CH}_{2}\right)_{7}-\mathrm{COOH}$. Undecane $\mathrm{C}_{11} \mathrm{H}_{24}$, an alkane hydrocarbon, was used as the dispersion medium - liquid carrier. The Undecane fluid MF-1 was synthesized in Ivanovo State Power Engineering University.

Figure 4 shows MF-column elastomagnetic parameters in the strong magnetic field which include oscillation frequency $v$, elasticity coefficients $k_{s}, k_{d}, k_{d \omega}$, $k_{p}$ and the correction for viscous fluid flow $\delta_{\eta}$ calculated using formulas (1), (2), (3), (4) depending on the magnetic field intensity gradient. The calculations were performed using the experimental results: by measuring the MF-column oscillation frequency; the data obtained by measuring the magnetic field intensity gradient at the MF-column base, static measurements of the water column level differences in the elbows of the U-tube $\Delta h$ and $\Delta z-$ MF-column displacement under hydrostatic pressure. In this case, the calculations of $k_{d}$ were performed using the values of $M_{x}$ belonging to the magnetization curve of the sample under study.

As it can be seen from the data shown in Figure 4, the elasticity coefficients $\mathrm{k}_{\mathrm{p}}, \mathrm{k}_{\mathrm{s}}$ and $\mathrm{k}_{\mathrm{d}}, \mathrm{k}_{\mathrm{d} \omega}$ corrected for the viscous fluid flow are approximately equal to each other to each other. Thus, we got an experimental confirmation of the above assumptions concerning the presence of equality, and we can obtain the value of the magnetization from two experiments:

magnetostatic saturation magnetization, which is obtained from (2) for the static displacement of the magnetic fluid horizontal column from equilibrium position.

$$
M_{S S}=\frac{\rho_{w} g}{2 \mu_{0}} \operatorname{tg} \alpha_{s}
$$

magnetodynamic saturation magnetization, which is obtained from (3) for magnetic fluid dynamic oscillations, taking into account viscous magnetic fluid flow.

$$
M_{S d}=\frac{2}{\mu_{0} \pi d^{2}} \operatorname{tg} \alpha_{d}
$$

To test the sensitivity of the proposed method to the change in the structure of the magnetic fluid, these fluids were modified. The sample MF-2 was partially evaporated from the sample MF-1. The physical parameters of the samples are shown in Table 2

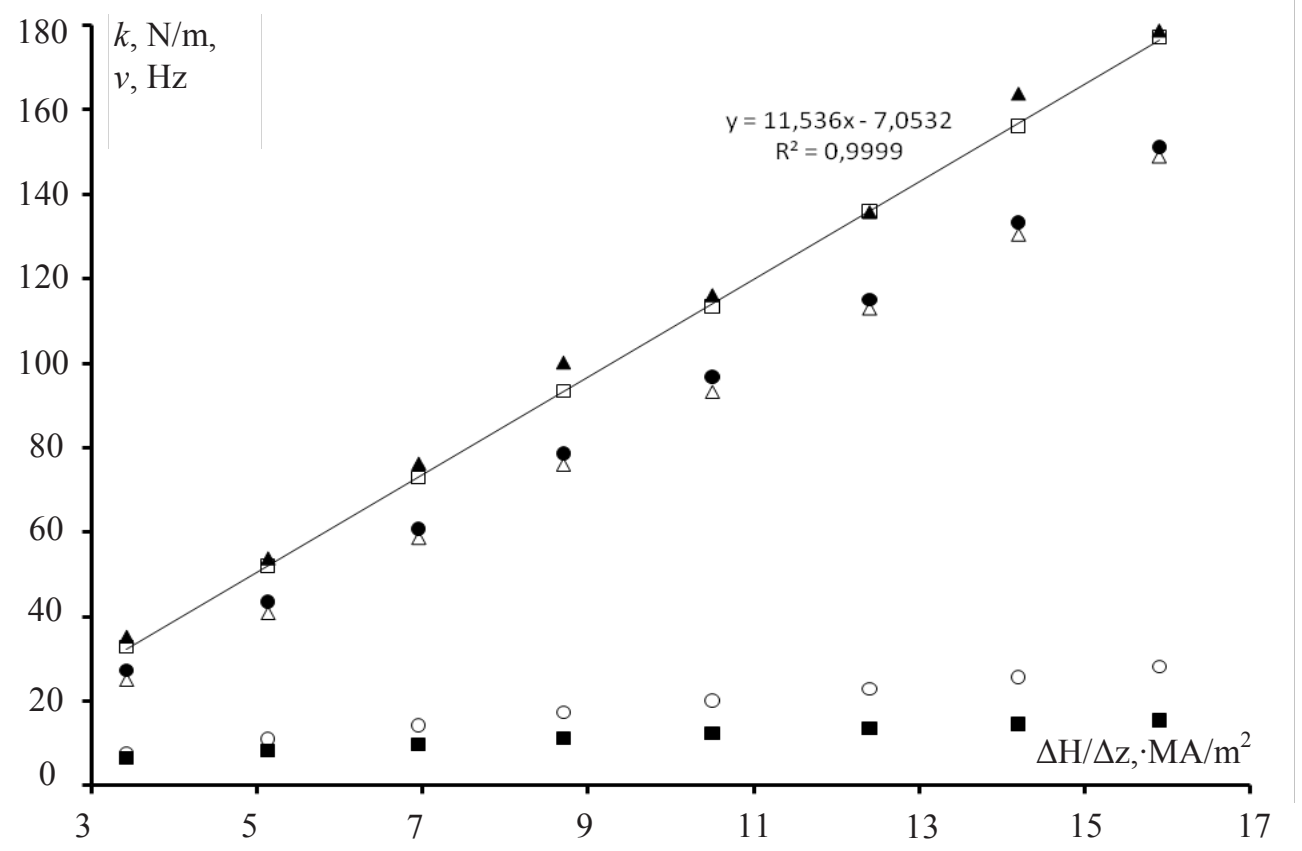

Figure 4. Parameters of oscillation system $\mathbf{\square}-v, \circ-\left|\delta_{\eta}\right|, \bullet-k_{d \omega}, \Delta-k_{d}, \square-k_{p}, \mathbf{\Delta}-k_{s}$ depending on the magnetic field intensity gradient 
Table 2

\begin{tabular}{|c|c|c|}
\hline Sample & MF-1 & MF-2 \\
\hline Carrier fluid & \multicolumn{2}{|c|}{ Undekan } \\
\hline Modification & & Evaporation of carrier fluid \\
\hline$\rho, \mathrm{kg} / \mathrm{m} 3$ & 1227 & 1240 \\
\hline$\varphi, \%$ & 10,8 & 11,1 \\
\hline$\chi$ & 1,6 & 2,1 \\
\hline $\mathrm{Ms}, \mathrm{kA} / \mathrm{m}$ & 40,4 & 45,1 \\
\hline$M_{S S,} \mathrm{kA} / \mathrm{m}$ & 40,9 & 46,2 \\
\hline$M_{S d}, \mathrm{kA} / \mathrm{m}$ & 40,6 & 45,8 \\
\hline
\end{tabular}

The magnetization curves obtained for liquids based on undecane are presented on the figure 5. The rhombus is a ballistic method, rectangles are the magnetodynamic method.

It can be seen that the data of the two methods are in good agreement. But for a structured magnetic fluid MF2 , the difference between the data of the two methods is greater. Data presented in Table 2 show that a small change in the nanoparticle volume fraction 'phi' (from 10.8 to $11.1 \%$ ) seems to produce an unproportionnally strong increase in saturation magnetization (from about 41 to about $46 \mathrm{kA} / \mathrm{m}$ ). This can be explained by the fact that when magnetic fluid is evaporated, a structure is formed in it.

\section{CONCLUSIONS}

The following results were obtained: the construction and validation of the model theory of elasticity formation of MF in a tube confined by magnetic levitation in a strong magnetic field were carried out, the described technique of complex measuring MF elasticomagnetic parameters in the strong magnetic field is of interest when studying magnetophoresis and aggregation of nanoparticles in magnetic colloids.

The research was carried out as a part of the project of state assignment of the Ministry of Education and Science of the Russian Federation. Project code: 3.2751.2017/PP.

\section{References}

1. Bogdan Sapinski. Of MR Fluids Recommended For Use In Shock Absorbers / Bogdan Sapinski, Wojciech Horak. Rheological Properties // acta mechanica et automatica, vol.7 no.2 (2013).p.107-110.

2. Bashtovoi, V.G. The effect of magnetophoresis and Brownian diffusion on the levitation of bodies in a magnetic fluid / Bashtovoi V.G., Polevikov V.K., Suprun A.E. // Magnetohdrodynamics. 2008. Vol. 44. №2. P. 121-126.

3. Boev M. L., Polunin V.M, P.A. Ryapolov, [et al.] Instability of the Flow of a Magnetic Liquid Pushing Down an Air Cavity // Russian Physics Journal. 2015. V. 57. №10. pp 1348-1355

4. Polunin V. Acoustics of nanodispersed magnetic fluids. New York- London: CRC Press, CISP, 2015. P. 472

5. Chechernikov V.I. Magnetic measurements. - M.: MGU, 1969. 387 p.

6. Polunin V. M., Ryapolov P. A., Platonov V. B., and Kuz'ko A. E. Free Oscillations of Magnetic Fluid in
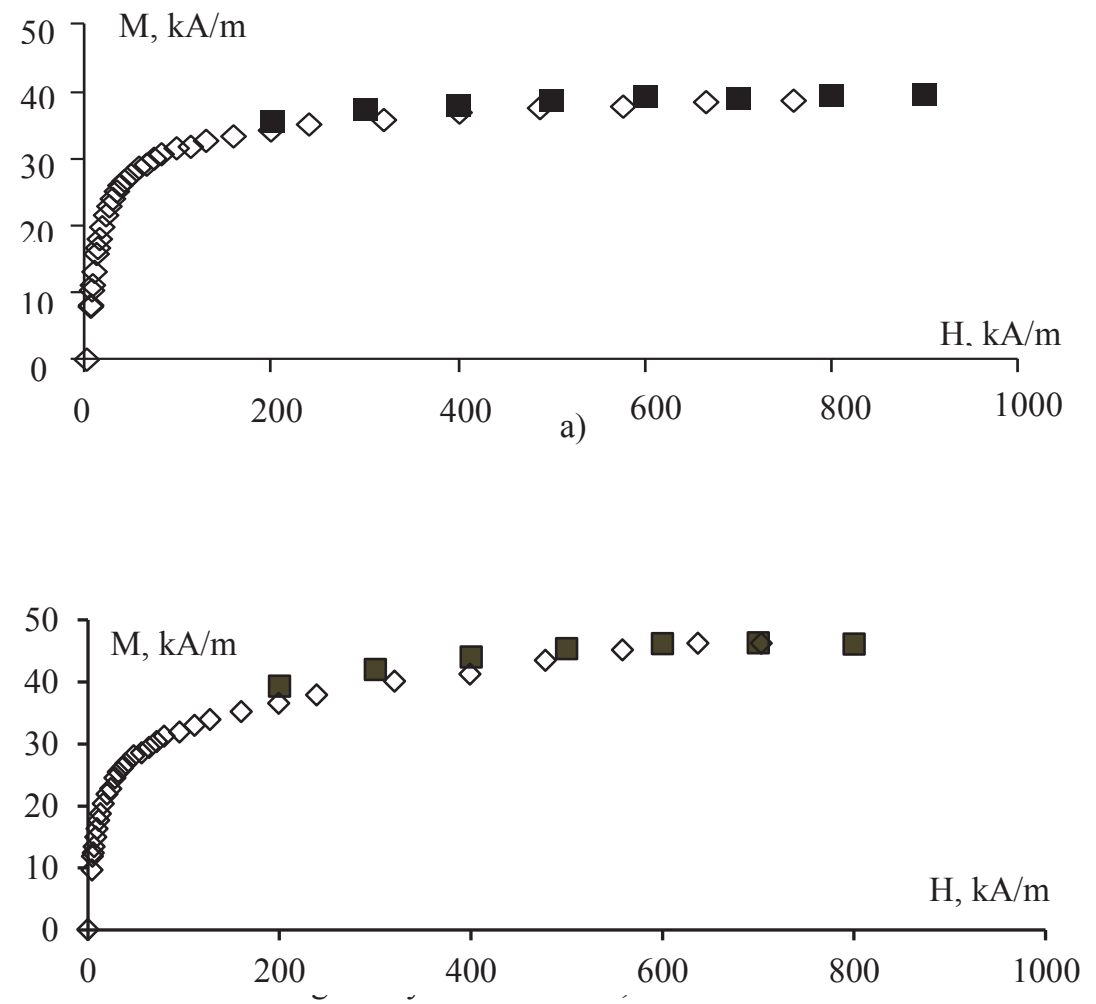
Strong Magnetic Field // Acoustical Physics, 2016, Vol.

62, No. 3, pp. 313-318. 